\title{
Bringing Social Media Apps in One Touch
}

\author{
Masoud Bandar Alsalman \\ *Faculty Member, Electrical Engineering Tech. Department, Industrial Training Institute - \\ Shuwaikh, PAAET, \\ Kuwait
}

\begin{abstract}
Nowadays, social networking is used in a wide range among a huge number of people worldwide, social media are not only used for pleasure in fact one of the greatest roles played in social media is for business-related projects, conferences, and meetings. In this proposed paper the main highlight issue linked is social media management. Although, there are hundreds of such applications that help in solving the problem of managing many social media accounts; yet, most of them varies with high irrelevant cost and supports unused features. Therefore, how about having a social media management application that is proposed to be able to solve management problems, supports social media users with the most desired features that will help to satisfy the recipient's demands and needs all in one platform.
\end{abstract}

Keywords : Communication, Mobile Applications, Social Media Applications.

\section{INTRODUCTION}

The internet is a worldwide system of computers that allows different users to get information from any other computers, but throughout the years the internet resolution grew faster and faster and smartphones were later on introduced to the world providing several applications to be built in one device. Mobile Applications are software applications that are established and created specifically for electronic mobile devices such as smart phones, tablets, etc [1]. Smartphones were the main key that opened the door of social media applications. Social media apps play a vital role around the world, people tend to socialize, connect, and communicate on daily basis; just by creating an account, users are able to post, share, and, face-time, with not only one, but with several other recipients at the same time. Nowadays, creating or introducing a new social media application is not difficult with the progression of technology and the enormous number of people depending on social media apps and using it on daily life routine, it is not difficult to create a new social application that performs and provides multiple tasks. The most popular apps used nowadays are Facebook, Instagram, LinkedIn, Skype, Snapchat, Twitter and WhatsApp, these applications allow users to add people manually by searching for the user's account name or number and also through scanning the pin code. Although mobile applications are considered to be very helpful, but like every other app, social applications have some cons, for instance, people spent more time moving from one application to another making it time wasting for the user, not only that, but it also consumes a lot of energy from the phones battery and a lot of space in the phones capacity which might later on slows down the phone use causing delays in the phone itself.

The objective of this paper is to introduce the OneSMApp which aims to group all the social media applications and bring them all under one umbrella. An app for both iOS and android users, that will categorize social media applications into different categories and will add up users instantly through a single touch, managing to bring all your preferred social media apps under one dashboard and a platform where accessing, posting, sharing pictures, information's, blogs and videos are easier and simpler.

The rest of this paper is organized as follows:

- Related work \& Motivation: in depth explanation of related applications to OneSMApp.

- Proposed framework and Solution: Optimized and simple way of communicating and using social media. A descriptive preview of the structure of oneSMApp. Combining all social media apps in one app.

- Statistics Analysis and performance evaluation: Simulated result of iOS and Android users. 


\section{RELATED WORK AND MOTIVATION}

This paper is of high importance and significance to anyone who owns a smart phone. It explains in considerable depth the solution and the functionality of the proposed problem. Since social media is being a daily job base for most of the people around the world therefore, acknowledging and understanding these seven applications and the tasks for them is critical:

\section{- Facebook:}

A social network that is used worldwide. It allows users to create profiles of themselves detailing their interests, hobbies, share pictures and enter private information, and finding a friend.

\section{- Instagram:}

This social network is a visual media platform. Where it allows accounts to upload images or short videos about their daily lives.

\section{- LinkedIn:}

This social network is designed for the business community. It is a professional way to help users to promote their personal brand virtually. It aims to allow registered members to document and to establish networks of people they personally trust and know.

\section{- $\quad$ Skype:}

This app allows users to make audio or video calls with people from all over the world. In addition, it provides a chatting service where messages can be exchanged between two different parties at two completely different locations.

\section{- Snapchat:}

This mobile app is designed to share photos, videos, drawings, and texts. The application differs from other applications by the way it shares its message where it disappears from the recipient's phone few seconds after being viewed.

\section{- Twitter:}

It is a micro blog service. It allows users to post short messages up to 280 characters. It is open for users to discuss what's happening about themselves or about others.

\section{- WhatsApp:}

This social networking app is the most popular messaging application that allows smartphones users to send messages. It is a texting service between smartphones that replaces the regular SMS text messages through the use of Internet.

Social media had developed the concept of communication by having an online content that is created by people and viewed by others. Its purpose is to share or read information and to support the human need for social interaction.

According to authors [2], the paper focuses on providing automation (automatically providing services) on the social media manager accountants. This app specifically works on only twitter. There are three main features that "Pokedem" is proposing which are; 1. Recommendations. 2. User profile. and 3. Analytics.

1. Recommendations: it is the main feature that this app is providing in which list of recommended account pops up on the dashboard, with an explanation of why should the user follow this account, where the account manager can accept, schedule or decline the offer.

2. User Profile: it provides the most followed and rich account based on the pokedem app itself which will also support recommendation.

3. Analytics: it provides analytics statistics and graph charts to show the number of liked tweets, retweets and who visited the account.

Therefore, such app could be a reference base to what the proposed project is, instead of having only twitter oneSMApp offers many similar social media applications integrated together inside one app.

In [3] the paper came to states the assignment of user classification to social media by using twitter. The articles discuss how did the authors came up with an idea to gather user's values of elements such as political orientation or ethnicity automatically and gain benefit from it in a recognizable way through their twitter feed. The paper's process work by applying a machine learning 
method from the information taken by the user, from that information they can first, report their result such as identifying ethnicity, and linking it to political subjects. Then their analysis will illustrate, how from these features them prove can be found.

In [4], this paper statistics and analytics were shown and proven that people worldwide download and use tourism applications on their smart phones when traveling, such a mobile application is trip advisor. Some of the applications used are not travel context related such as the social media applications (Google Maps, Twitter, Facebook) yet users tend to use such social applications for the purpose of communication and sharing pictures, videos etc. This research paper is based on categorizing and classifying different taxonomies of tourism apps after analyzing them and gathering them into several lists such lists were the navigation, social, mobile marketing, security and emergency, entertainment, information and transactional. Each of these categories had a sub list where its shows the linked mobile applications to the category.

According to the authors of [5] paper, it talks about the ethical implications and risks of using automated system that learn from social media data, missing or Erroneous Data for those who are not using hashtag to collect data, the solution may be that "use content analysis and synonyms for gathering content allow for more flexibility and wider coverage". Validation of data labels regarding for the high variety of topics and domains discussed on social media. Flawed interpretation of results such when the system interpretation of data be presented in a graphical form, thus potentially distracting the user from whether the data is misrepresented.

The idea of creating social media management application varied from different users, where several developers implemented this idea into a more enhanced application of these management applications have similar features and distributes the same algorithm which are built in and implemented in the apps. Making these features the most used and reliable features that satisfies the consumer's needs.

\section{PROPOSED FRAMEWORK}

Managing multiple of social media account is tiring and time consuming in terms of sharing a certain picture to many different applications or whether adding a friend in a certain category. Thus, this app is trying to solve and provides users with what the most demanded and reliable features that each and every social media app user truly wants.

As social media applications grew smarter, it allowed a "one to many communication". Such as Instagram, where it provided a feature that allows users to post a photo in the application itself and be able to choose other applications such as Facebook, Twitter, and Tumblr that the user would also like to post in. The ability to have a one to many communications is a far smart feature.

Although many different social media application management allows the users with scheduling feature, yet this algorithm is only applied to certain apps for example Hootsuite [6], where it allows scheduling for tweets in twitter apps if the user prefers to post the tweet in different timing.

Prioritizing (user interest) is the simplest way to satisfy the consumer needs when it comes to viewing their most favored topics among other people. The idea of prioritizing comes in many different forms whether its for parametrized priority or content priority. With parameterized priority it is done through analyzing the following: Number of views, number of likes, number of followers and etc.; therefore, it basically means that it depends by a number and is somehow written and executed with Hard code algorithm. However, when considering the content prioritizing is when the user specifies a certain word or field and targets that data to be shown and read at the top of everything. Moreover, content user interest is implemented through machine learning algorithm.

Instagram and Twitter implement notification and pining panel in which users can be noted whenever the person they pinned have shared a post. This feature notify certain people posts in one's application before viewing others and informing the user whenever the person they have pinned has posted anything, thereby, keeping other people post in track. Hootsuite [6], allows users to also pin an account to get notified whenever the user has posted anything from all different applications in the app itself, thereby, providing a better access to other users post in a fast and enhanced way.

OneSMApp delivers a one to many communications where all social media applications are combined together and users can share comments, videos, and photos in all social media applications all together in this app however it provides more variety of app choices. OneSMApp is also trying to propose scheduling based upon time zones, such a case if a user wants to share a picture into three different applications Instagram, Twitter, and Facebook, the user can schedule posting this picture from their current 
time into the desired time zone they prefer. OneSMApp is proposing to solve and have all the features and implementing them in an easy and more improved way.

\section{iV. Performance Evaluation}

Descriptive statistics provide an idea about the average and standard deviation for each variable.

- Number of applications vary between 2 and 5 applications per user with an average of 4.0 (Median). Most users (60\%) have 4 applications on their mobile phones.

Table No. 1 number of applications and it's percentage.

\begin{tabular}{|c|c|c|}
\hline Number of Apps & Count & Percentage \\
\hline 2 & 2 & 1.67 \\
\hline 3 & 13 & 10.83 \\
\hline 4 & 72 & 60.00 \\
\hline 5 & 30 & 25.00 \\
\hline 6 & 3 & 2.50 \\
\hline
\end{tabular}

Where $\mathrm{N}=120$ summation of the number of users.

- Average daily time of using mobile phone per user is 396.3 minutes (6.6 hours). Almost Half of this time in the Evening (average of 197.9 minutes), 31.5\% of this time in the Afternoon (average of 125.25 minutes) and 18.5\% (average of 73.16 minutes) of this time in the morning.

- Frequency of daily visits per user to these applications vary between 4 times and a day and a maximum of 172 visits a day with an average of 40 visits a day.

- Average daily Storage of each user from these applications is 5.1 GB.

- Average delay time is 10.4 seconds for an average of 4 applications for each user.

For all Other parameters, IOS is higher. These difference between IOS and Android can be highlighted if needed similar to the above.

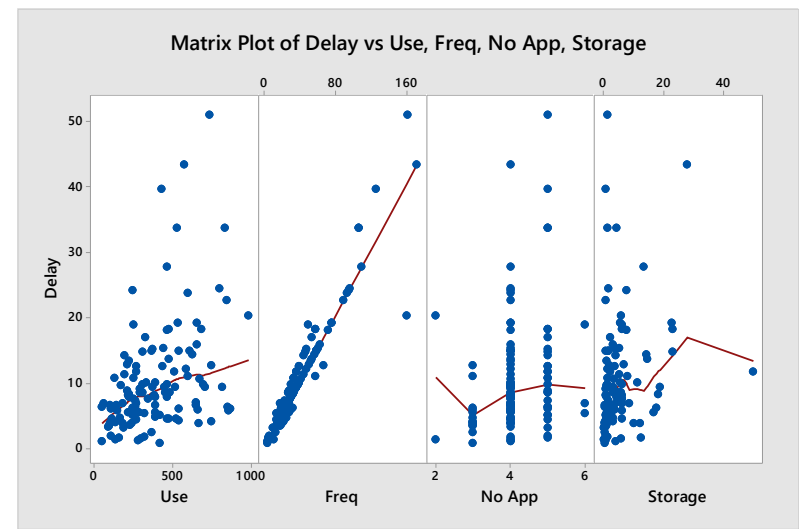

Fig 1 Matrix plot of all the variables

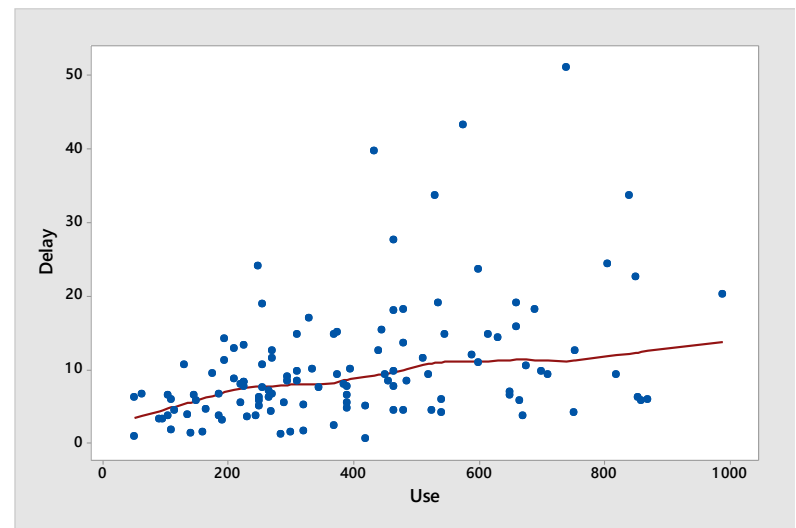

Fig 2 Delay in seconds vs number of use in minutes 


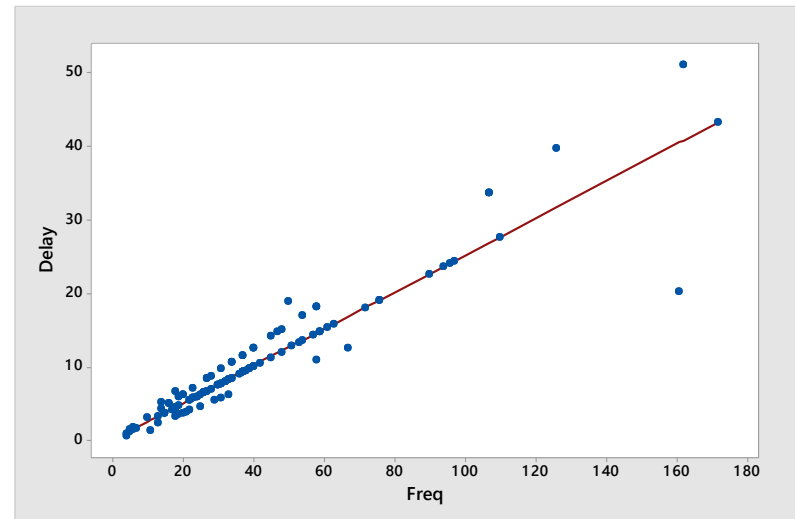

Fig 3. Delay in seconds vs Frequency

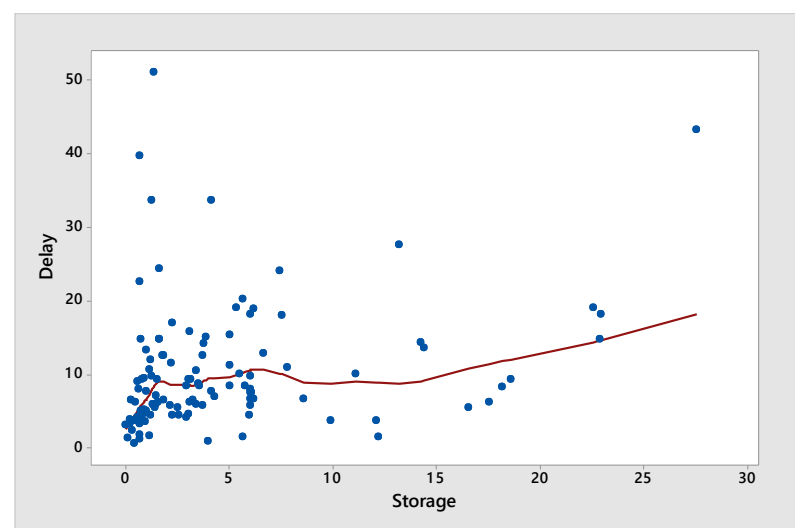

Fig 4 Delay in seconds vs Storage Capacity

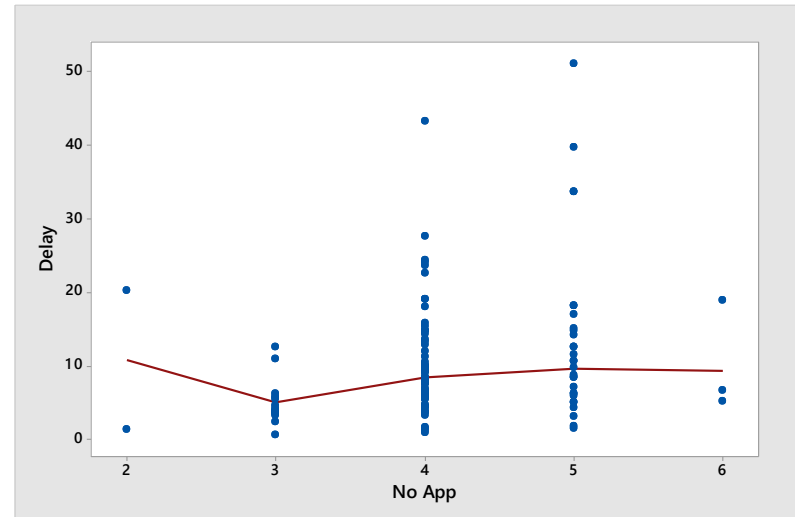

Fig 5 Delay in seconds vs Number of Applications

\section{CONClusion}

The application idea was supported by having a questioner that was distributed to both iOS and Android users where it examined seven social media applications in terms of minutes spent per day, delay caused by switching between those applications and the frequency which is measured by the number of times the user accessed the applications per time slot, and power and storage consumption. Secondly, to gather an accurate and considerable data statistic the results of the questioner were simulated proving the effectiveness of the proposed solution in enhancing the disadvantages of managing social media applications. Moreover, this IEEE paper has been proposed introducing the problem that were studied, all the proposed solution, related work, and the plans that can be added to the application in the future. 


\section{REFERENCES}

[1] What is mobile application management (MAM)? - Definition from WhatIs.com. (n.d.). Retrieved December 07, 2017, from http://searchmobilecomputing.techtarget.com/definition/mobile-application-management-MAM

[2] Corcoglioniti, F., \& Giuliano, C. Pokedem: an Automatic Social Media Management Application. Proceedings of the Eleventh ACM Conference on Recommender Systems -RecSys 17. Ovember 9, 2017

[3] A Machine Learning Approach to Twitter User Classification. (n.d.). Retrieved November 11, 2017, from http://ieeexplore.ieee.org/abstract/document/7959042/

[4] Eden, H., \& Gretzel, U. A Taxonomy of Mobile Application in Tourism, 2012, 47-50, University of Wollongong, https://ro.uow.edu.au/commpapers/2510

[5] Lanfranchi, V. (2017, May). Machine Learning and Social Media in Crisis Management: Agility v/s Ethics. Conference: ISCRAM 2017, May 2017

[6] “The Top 15 Tools for Managing Social Media Accounts,” Search Engine Journal, 11-Oct-2017. 